\title{
Cumplimiento sobre las recomendaciones de uso y evaluación del impacto económico de un programa de uso restringido de imipenem-cilastatina
}

\author{
Alberto Fica C., Mario Luppi M., Roberto Olivares C., Lorena Brito F., Isabel Zilleruelo L. y Lorena Muñoz C.
}

\section{Imipenem-cilastatin use in a university hospital in Chile}

Imipenem is an expensive broad-spectrum antimicrobial, reserved for infections caused by multi-resistant nosocomial pathogens. Since 2001 our university hospital applies a restriction policy that allows rejecting or authorizing its use after a supervising evaluation whith pre-specified criteria for appropriate or inappropriate use. An audit was performed for all the supervisions made during the periods of March-April and SeptemberOctober, 2004, totalizing 136 treatments. In global terms, 58.1\% of treatments were considered appropriate and $11.8 \%$ inappropriate; other $20.6 \%$ had been discontinued by physicians in charge prior to evaluation. Susceptibility to other antimicrobials compounds was the main reason for inappropriate use. The remaining fraction involved deceased or discharged patients. Discontinuation of treatments by supervising physicians allowed to save 75 days and 362 vials of imipenem equivalent to US $\$ 6,777$ during this period after discounting administrative and human resources costs.

Key words: Imipenem, antibiotic restriction policy, active surveillance, economical impact.

Palabras claves: Imipenem, restricción de compuestos antimicrobianos, supervisión activa, impacto económico.

\section{Introducción}

$\mathrm{M}$ icroorganismos que provocaban infecciones otrora fácilmente controlables, han emergido como problemas de resistencia antimicrobiana y han limitado las opciones terapéuticas, afectando también las posibilidades de curación o mejoría clínica. En Chile se ha documentado progresivamente la resistencia antimicrobiana en ambientes comunitarios que involucra aislados de Escherichia coli asociados a infección urinaria, Neisseria gonorrhoeae o Streptococcus pneumoniae resistente a penicilina, cepas de Haemophilus influenzae o Moraxella catarrhalis resistentes a amoxicilina y aislados de Shigella resistentes a diferentes compuestos ${ }^{1-6}$.

La situación es más desastrosa en ambientes nosocomiales, siendo frecuente o progresiva en Chile la presencia de enterobacterias portadoras de $\beta$ lactamasas de espectro extendido, Pseudomonas aeruginosa hiperproductoras de $\beta$-lactamasas cromosomales y Staphylococcus aureus resistente a cloxacilina o Enterococcus resistente a vancomicina ${ }^{7-9}$.

Para contener la emergencia de la resistencia antimicrobiana en la comunidad y en el hospital, se han delineado diferentes estrategias de intervención y que incluyen capacitación médica, restricción al uso de antimicrobianos, autorización previa, remoción de compuestos, formularios de solicitud, detenciones automáticas, educación de los pacientes, rotación cíclica de compuestos, desarrollo de guías clínicas y la omisión de susceptibilidades específicas en el informe de laboratorio ${ }^{10-13}$

Imipenem-cilastatina es un compuesto de amplio espectro, baja toxicidad y alto costo, que se reserva estratégicamente para infecciones por bacterias multiresistentes debido a su estabilidad a una diversidad de $\beta$-lactamasas presentes en las bacterias de los hospitales. Sin embargo, su uso ha estado asociado a la emergencia de cepas resistentes a este compuesto y a la pérdida de alternativas terapéuticas ${ }^{14}$.

El año 2001 se inició en el Hospital Clínico de la Universidad de Chile un programa de uso controlado de imipenem-cilastatina con el objetivo de promover el uso racional de este antimicrobiano, reducir la presión selectiva sobre bacilos gramnegativos resistentes y contener costos. Bajo este programa, el uso de imipenem-cilastatina es restringido, debiendo su continuación ser autorizada por alguno de los médicos especialistas en enfermedades infecciosas. Los criterios de uso apropiado se han aplicado sin variación
Hospital Clínico

Universidad de Chile

Comité de Infecciones

Intrahospitalarias (AFC)

Sección de Infectología (ALC, MLM, ROC)

Servicio de Farmacia (LBF)

Unidad de Medicamentos, Depto. Abastecimiento (IZL, LMC)

Recibido: 7 noviembre 2005 Aceptado: 28 junio 2006

Correspondencia a: Alberto Fica Cubillos afica@redclinicauchile.cl 
desde el año 2001, se han divulgado en actividades de capacitación y han estado disponibles como capítulos específicos en diferentes ediciones del manual local de Prevención y Control de Infecciones Intrahospitalarias y en la página web del hospital.

El programa de control sobre imipenem-cilastatina se engloba en una estrategia global que involucra otros antimicrobianos tales como vancomicina, ertapenem, ganciclovir, anfotericina $\mathrm{B}$ deoxicolato $\mathrm{y}$ caspofungina y que se ha expandido en forma continua. El impacto para algunos de estos compuestos, particularmente vancomicina y ertapenem, ha sido previamente evaluado y divulgado en informes internos. En el caso de vancomicina, los resultados han sido también presentados en un congreso local por los autores ${ }^{15}$

El objetivo del presente trabajo fue evaluar el grado de cumplimiento de las recomendaciones publicadas sobre la prescripción de imipenem-cilastatina, evaluar el programa de control en su capacidad para detectar tratamientos inapropiados y suspenderlos y, finalmente, estimar el impacto económico del ahorro.

\section{Metodología}

Escenario. Hospital universitario de nivel terciario con 570 camas.

Diseño. Revisión de pautas de supervisión utilizadas en los pacientes en los cuales se inició un tratamiento con imipenem-cilastatina durante dos períodos del año 2004 (marzo-abril y septiembre-octubre). Se analizó la totalidad de los pacientes que recibieron este compuesto durante el período.

Programa de supervisión. Este programa se activa con el aviso de farmacia cada vez que a un paciente se le indica imipenem-cilastatina. Los pacientes son visitados por uno de los médicos especialistas en enfermedades infecciosas del hospital, el que aplica una pauta estructurada y decide aprobar, rechazar este compuesto, o visitar más adelante al paciente (máximo 5 días desde el inicio) para nuevamente aprobar o rechazar este antimicrobiano. La pauta no puede ser dejada en blanco y debe retornar al Servicio de Farmacia y considera situaciones de alta, traslado o muerte del paciente. Ante el aviso de no aprobación por parte de los médicos supervisores, el Servicio de Farmacia, bloquea la entrega del compuesto. El programa fue iniciado el año 2001 y la autorización para interrumpir los tratamientos fue otorgada por el Director del Hospital. Las pautas de supervisión para cada paciente visitado son archivadas posteriormente.

Criterios de uso inapropiado y razones para la suspensión del tratamiento. Los criterios de uso in- apropiado vigentes durante el desarrollo de este programa se presentan en el anexo 1. Estos criterios fueron elaborados en forma arbitraria, localmente, y básicamente consideran inapropiado el uso de este compuesto cuando hay otras alternativas o si se trata del manejo de patología adquirida en la comunidad. Las causas de suspensión se desglosan en detalle en la sección resultados. Se debe mencionar que el uso empírico de imipenem-cilastatina no está prohibido en nuestro hospital excepto si se cumplen algunos de los criterios del anexo 1. Ello porque en diversas situaciones de la atención hospitalaria no se puede postergar una decisión terapéutica a la espera de la información microbiológica.

Evaluación del impacto económico. La evaluación de impacto económico se realizó de acuerdo a los frascos totales ahorrados hasta la fecha de suspensión del tratamiento alternativo indicado, hasta el fallecimiento del paciente o hasta el reinicio de imipenemcilastatina. En estos cálculos se consideró la dosis y duración exacta del tratamiento con imipenemcilastatina. Luego de obtener los valores para frascos de tratamiento ahorrado, se descontó el costo del tratamiento alternativo indicado cuando éste fue utilizado y los costos administrativos y en recursos humanos utilizados por el programa.

Dosis diaria definida (DDD) por 100 dias camas ocupadas. Se utilizó este indicador para evaluar la densidad de uso de tratamientos en los meses analizados en comparación a los meses no evaluados. El cálculo se efectúa de acuerdo al índice ocupacional con DDD por 100 días camas ocupadas. Se consideró una dosis estándar de 2 gramos al día de imipenemcilastatina.

\section{Resultados}

\section{Primera visita de supervisión}

La supervisión inicial se realizó a los 1,95 días promedio desde el inicio del tratamiento. Un total de 67 tratamientos fue analizado para los meses de marzo y abril y 69 en el segundo período (total 136). Estos tratamientos quedaron desglosados en la primera visita en 5 categorías, (Figura 1) y corresponden a:

- tratamientos empíricos iniciales (requieren una nueva visita)

- uso apropiado (aprobados)

- uso inapropiado (rechazado)

- tratamientos ya suspendidos por el tratante

- otras condiciones tales como pacientes fallecidos, altas o traslados. 
En la primera visita de supervisión se detectó aproximadamente un tercio de tratamientos apropiados $(\mathrm{n}=47 ; 34,6 \%)$ y un alto porcentaje quedó programado para una segunda visita de supervisión $(n=54 ; 39,7 \%)$. En esta primera instancia, 5,1\% $(n=7)$ de los tratamientos fueron considerados inapropiados y suspendidos y un porcentaje no despreciable ya había sido suspendido por los propios médicos tratantes $(n=19 ; 14 \%)$.

\section{Segunda visita de supervisión}

Ella se realizó en promedio a los 3,3 días después de la primera visita. Su objetivo fue mantener una supervisión adecuada sobre aquellos casos que no pudieron ser resueltos en la primera oportunidad, habitualmente por encontrarse cultivos pendientes. En esta segunda visita se analizaron todos los casos programados (Figura 2).

En esta oportunidad se aprobó el antimicrobiano en la mayor parte de los casos $(59,3 \%)$, aunque se detectó un número importante de usos inapropiados $(n=9$; $16,7 \%)$. Al igual que en la primera visita, en una fracción de los casos el compuesto ya había sido suspendido por el propio médico tratante $(n=9 ; 16,7 \%)$.

\section{Resultados globales}

En la Tabla 1 se presentan las categorías finales observadas en los 136 tratamientos bajo supervisión. En términos globales, casi $60 \%$ de los casos estuvo asociado al uso adecuado de este compuesto $(n=79)$ y sólo 16 casos fueron catalogados como inapropiados $(11,8 \%) \mathrm{y}$, en consecuencia, suspendidos los tratamientos.

Tabla 1. Resultados globales de la supervisión

de 136 tratamientos de imipenem-cilastatina durante cuatro meses. Hospital Clínico Universidad de Chile, 2004

\begin{tabular}{|lcr|} 
Categoría final & n & \multicolumn{1}{c|}{$\%$} \\
\hline Tratamiento apropiado & 79 & 58,1 \\
\hline Ya suspendido por médico tratante & 28 & 20,6 \\
\hline Inapropiado & 16 & 11,8 \\
Fallecidos y otros & 13 & 9,6 \\
\hline Total & 136 & 100,0 \\
\hline
\end{tabular}

En la mayor parte de las suspensiones por tratamiento inapropiados estuvieron involucrados microorganismos susceptibles a otros antimicrobianos (Tabla 2).

\section{Suspensiones de tratamiento por usos inapropiados}

Sólo en 11 de los 16 tratamientos inapropiados hubo una interrupción real del tratamiento $(68,7 \%)$. En 4 no hubo suspensión, 2 por una descoordinación con el Servicio de Farmacia lo que llevó a mantener el tratamiento y en los otros 2 casos hubo fallas por parte de los médicos supervisores para suspenderlos efectivamente. En un caso adicional, la interrupción fue efectuada el mismo día que el paciente falleció.

Las dosis diarias de imipenem-cilastatina en los tratamientos suspendidos variaron de $500 \mathrm{mg}$ a 4 gramos por día, aunque en la mayor parte de los casos fue de 2 gramos diarios (datos no mostrados).

El detalle de los tratamientos alternativos utilizados en los casos de interrupción del compuesto carbapenémico se presenta en la Tabla 3. En ella se puede observar una gran dispersión de esquemas y duraciones de tratamiento. Desde esquemas tetra-asociados a

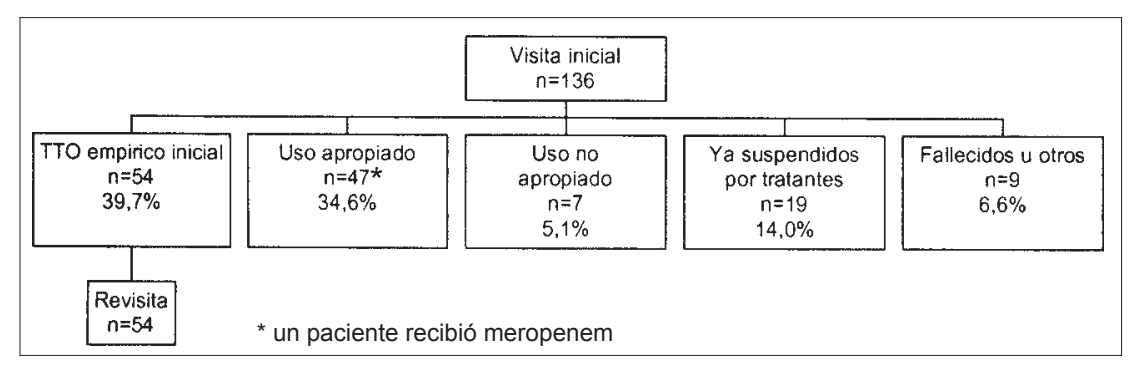

Figura 1. Distribución de la primera visita en 136 supervisiones sobre el uso de imipenem-cilastatina en dos períodos diferentes. Hospital Clínico Universidad de Chile, año 2004.

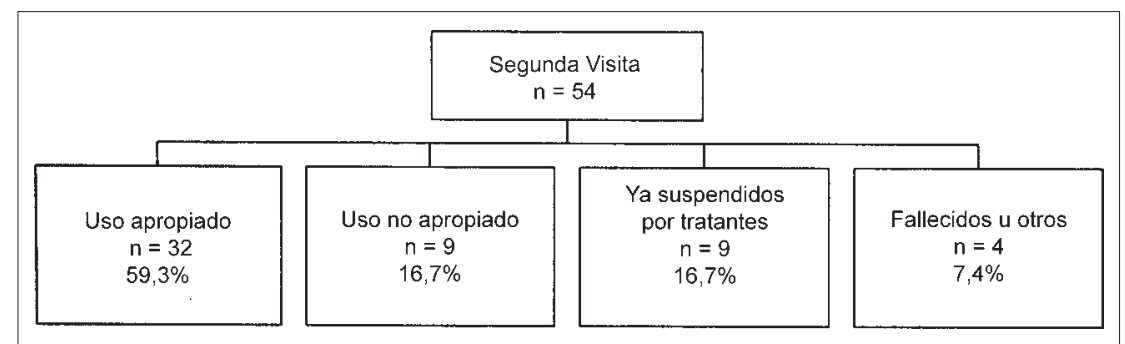

Figura 2. Distribución de resultados de la segunda visita de supervisión para 54 pacientes bajo tratamiento con imipenem-cilastatina. Hospital Clínico Universidad de Chile, año 2004.

\begin{tabular}{|lcc|}
\hline $\begin{array}{c}\text { Tabla 2. Causas de suspensión de tratamientos inadecuados con } \\
\text { imipenem-cilastatina durante } 4 \text { meses de supervisión activa. } \\
\text { Hospital Clínico Universidad de Chile, año }\end{array}$ \\
\hline Causa & n & $\%$ \\
\hline Microorganismos susceptibles a otros antimicrobianos & 11 & $68,8 \%$ \\
\hline Cultivos negativos en sitios de infección & 4 & $25,0 \%$ \\
\hline Tratamiento de paciente terminal sin infección identificada & 1 & $6,3 \%$ \\
\hline Total & 16 & $100 \%$ \\
\hline
\end{tabular}


monoterapias y duraciones de 2 a 11 días. En un caso no se usó ningún tratamiento opcional. Los esquemas con ceftazidima, sulbactam/ampicilina o sulbactam/ cefoperazona, con o sin amikacina, condensan estas opciones. Los frascos ahorrados de imipenem-cilastatina variaron entre 8 y 88 unidades de $500 \mathrm{mg}$ en estas 11 interrupciones (Tabla 3)

La evolución de los pacientes con interrupción del tratamiento fue favorable en 8 casos, aunque en 2 de ellos fue necesario un cambio de esquema a los 5 y 7 días por sobreinfección con otro agente resistente al esquema en uso (Tabla 3). Los 3 pacientes restantes fallecieron, incluyendo 2 casos por cáncer terminal. En un caso el paciente falleció por falla multiorgánica e infección del torrente sanguíneo por Acinetobacter baumannii, sin haber tenido respuesta con imipenemcilastatina previamente o con el esquema alternativo (Tabla 3).

\section{Evaluación económica de las interrupciones de tratamiento}

Los 11 tratamientos efectivamente interrumpidos significaron un total acumulado de 75 días de tratamiento suspendido (Tabla 3). Este valor se obtuvo considerando los días que el compuesto no se usó hasta que el tratamiento alternativo terminó $(9$ de 11

\section{Tabla 3. Tratamientos alternativos utilizados ante 11 interrupciones de imipenem-cilastatina}

\section{Esquema alternativo}

Nebulizaciones con amikacina $400 \mathrm{mg} / 8 \mathrm{~h}$ + ceftazidima $1 \mathrm{~g}$ c/8 ev +amikacina $400 \mathrm{mg} \mathrm{c} / 8 \mathrm{ev}$ +cloxacilina $2 \mathrm{~g}$ c/6 ev Ceftazidima $1 \mathrm{~g} \mathrm{c} / 8 \mathrm{ev}$ Sulbactam/ampicilina 1 fco c/8 ev Ceftazidima $1 \mathrm{~g}$ c/8 + amikacina $500 \mathrm{mg} / \mathrm{d}$ ía ev

Sulbactam/ampicilina 1 fco c/6h ev (días)

11 de imipenem

10

9

2

20

Sulbactam/cefoperazona

2 fcos c/12+ amikacina $1 \mathrm{gr} / \mathrm{día} \mathrm{ev}$

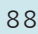

8

40

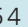

8

5

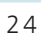

0

Diagnóstico, agente causal y evolución

Fibrosis quística

Bronquitis por $P$. aeruginosa multisensible

Evolución favorable y alta

Cáncer terminal de tiroides

Sepsis grave sin agente aislado y sin respuesta a 4 días de imipenem-cilastatina. Fallece por cáncer

Bronquitis asociada a ventilación mecánica por $A$. baumannii sensible a otras alternativas. Evolución favorable

Shock séptico post-quirúrgico abdominal e infección del torrente sanguíneo por $A$. baumannii. Sin respuesta a 5 días de imipenem-cilastatina. Fallece por falla multiorgánica

Granulomatosis de Wegener, inmunosupresión. Bacteriemia y neumonía por $A$. baumannii sensible a otras alternativas. Evolución favorable por 5 días hasta sobreinfección pulmonar por $P$. aeruginosa multiresistente

Pancreatitis aguda complicada con colecciones abdominales por $P$. aeruginosa sensible a otras alternativas. Evolución favorable por 7 días hasta sobreinfección por Enterobacter multiresistente

Ceftazidima $1 \mathrm{~g} \mathrm{c} / 8 \mathrm{ev}$ 7 211 Sulbactam/ampicilina $2 \mathrm{fcos} \mathrm{c} / 6 \mathrm{~h}$ por 2 días, luego sulbactam-cefoperazona

$2 \mathrm{f} \cos \mathrm{c} / 12+$ amikacina $1 \mathrm{~g} / \mathrm{dí}$ ev

$$
\text { Sulperazona } 2 \mathrm{fcos} c / 12
$$$$
\text { Amikacina } 500 \text { mg/d ev }
$$$$
\text { Sin esquema alternativo }
$$$$
\text { Total }
$$

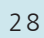

52
Leucemia mieloide aguda. Neutropenia febril. Bacteriemia por $P$. aeruginosa. Evolución favorable

Sepsis post-quirúrgica abdominal. Bacteriemia por A. baumannii con evolución favorable
Peritonitis post-quirúrgica con cultivos positivos a $P$. aeruginosa y E. coli. Evolución favorable.

Accidente cerebrovascular reciente, ITU sintomática por E. coli multiresistente. Evolución favorable

Tumor mediastínico, hemotórax. No se identificó microorganismo. Fallece por cáncer terminal. 
casos), se reinició imipenem-cilastatina (un caso) o el paciente falleció durante el tratamiento (un caso). De la misma manera, los frascos ahorrados fueron calculados a las dosis reales que el paciente utilizaba. Este parámetro señaló un ahorro de 362 frascos en el período.

Para el período de supervisión, el ahorro en gasto no incurrido alcanzó los 5.600.140 pesos y un ahorro neto de 4.147.085 pesos luego de descontar el costo de los tratamientos alternativos usados. El ahorro final al considerar tanto los gastos en recursos humanos como los costos administrativos fue de 3.524 .133 pesos (US 6,777).

Dosis diaria definida durante períodos bajo análisis. La auditoria de los tratamientos supervisados se efectuó durante un período en el que se usó en promedio mensual menos DDD por días camas ocupadas de imipenem-cilastatina en comparación a los 8 meses restante (9,5 vs 14,9 DDD-100 días camas ocupadas). Esto indica que los ahorros de tratamiento logrados en los meses bajo supervisión fueron obtenidos en épocas con una menor densidad de uso de este compuesto y que cualquiera estimación anual tiende a subestimar el ahorro neto total.

\section{Estimación del ahorro anual}

Teniendo en cuenta que la supervisión se efectúa durante 11 de los 12 meses del año, la proyección de ahorro en frascos del fármaco alcanza 995 unidades,
15.392.650 de pesos anuales en facturación al hospital por el proveedor y un ahorro real de 11.404 .483 pesos al considerar el costo de los tratamientos alternativos usados (Tabla 4). El ahorro real puede estimarse en 9.691.366 pesos al incluir los gastos administrativos y de recursos humanos del programa. Para los cálculos del compuesto se estimó un precio por frasco de 15.470 pesos durante el año 2004 (IVA incluido), incluyendo el SF viaflex ${ }^{\circledR}$ (dispositivo desechable que contiene solución salina estéril empleada en la dilución del fármaco).

\section{Distribución de tratamientos aprobados, suspendidos o ya suspendidos por los propios médicos tratantes en diferentes unidades}

En la Tabla 5 se presentan los resultados de los tratamientos aprobados y suspendidos (por supervisión) para aquellos servicios o unidades con mayor frecuencia de uso de este compuesto. Los resultados se presentan en orden decreciente de acuerdo al volumen de tratamientos usados. Las ocho dependencias incluidas representan el $82,3 \%$ de los tratamientos indicados y supervisados en el período

Se puede observar que la mayor frecuencia de tratamientos suspendidos no se correlaciona con las unidades que más utilizan este compuesto. La lista de usos suspendidos la encabeza la Unidad Coronaria, seguida de Intermedio Quirúrgico y en tercer lugar la UCI.

Tabla 4. Evaluación económica de las interrupciones de tratamiento de imipenem-cilastatina durante 4 meses de supervisión activa. Hospital Clínico Universidad de Chile, año 2004

\section{Parámetro}

Tratamientos suspendidos $n$

Tratamiento efectivamente interrumpidos ( $n$ )

Días ahorrados de tratamiento (días)

Frascos ahorrados (n)

Costo no incurrido para el período

Ahorro neto en tratamiento en 4 meses luego de descontar el costo de los tratamientos alternativos usados

Costos administrativos y de recursos humanos*

Ahorro real
Resultados

16

11

75

362

5.600 .140 pesos

4.147.085 pesos

622.952 pesos

3.524 .133 pesos

Estimación de ahorro anual

Frascos ahorrados en un año

$\sim 995$

Costo no incurrido de imipenem-cilastatina para un año

15.392 .650 pesos

Ahorro neto en tratamiento para 11 meses de supervisión obtenidos al descontar costo de tratamientos alternativos

Ahorro neto final al incluir gastos administrativos y de recursos humanos

$\sim 11.404 .483$ pesos

9.691.366 pesos

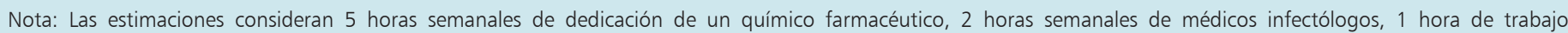

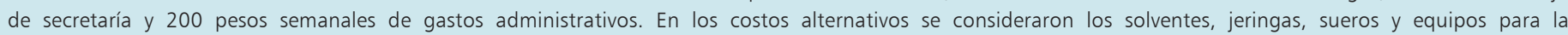
administración, por estar comparándose con imipenem-cilastatina monovial con SF viaflex, incluido en el costo. 
Tabla 5. Distribución de tratamientos aprobados y suspendidos de imipenem-cilastatina en unidades con mayor frecuencia de uso durante 4 meses de supervisión activa. Hospital Clínico Universidad de Chile, 2004

\begin{tabular}{|c|c|c|c|c|c|}
\hline Servicio o Sección & Total tratamientos & Tratamientos aprobados & $\%$ aprobados & Tratamientos suspendidos & $\%$ no aprobados \\
\hline $\mathrm{UCl}$ & 31 & 17 & 54,8 & 6 & 19,4 \\
\hline UHO & 15 & 9 & 60,0 & 1 & 6,7 \\
\hline UNI-Nefrología & 14 & 8 & 57,1 & 0 & 0,0 \\
\hline U. Coronaria & 13 & 5 & 38,5 & 3 & 23,1 \\
\hline Intermedio Médico & 13 & 7 & 53,8 & 1 & 7,7 \\
\hline Intermedio Quirúrgico & 11 & 7 & 63,6 & 2 & 18,2 \\
\hline Medicina & 11 & 6 & 54,5 & 1 & 9,1 \\
\hline Cirugía & 5 & 4 & 80,0 & 0 & 0,0 \\
\hline Total & 112 & 62 & & 14 & \\
\hline
\end{tabular}

Tabla 6. Total de tratamientos aprobados y ya suspendidos por médicos tratantes en servicios con mayor frecuencia de uso durante 4 meses de supervisión activa. Hospital Clínico Universidad de Chile

\begin{tabular}{lcc} 
Servicio o Sección & $\begin{array}{c}\text { Total tratamientos aprobados }+ \\
\text { ya suspendidos por tratantes }\end{array}$ & $\%$ \\
\hline Medicina & 10 & 90,9 \\
UHO & 13 & 86,7 \\
UNI-Nefrología & 12 & 85,7 \\
Cirugía & 4 & 80,0 \\
UCl & 23 & 74,2 \\
Intermedio Quirúrgico & 8 & 72,7 \\
\hline Intermedio Médico & 9 & 69,2 \\
U. Coronaria & 8 & 61,5 \\
\hline
\end{tabular}

\section{Otros aspectos}

La suma de tratamientos aprobados y de aquellos ya suspendidos por los propios médicos tratantes, representa para un Servicio Clínico un potencial indicador de calidad del uso de este compuesto. Un resumen de este indicador se presenta en la Tabla 6 para las unidades de mayor uso. Destacan con mejores porcentajes las unidades o secciones de Medicina, Unidad de Hematología Oncológica, Unidad Nefrológica Intensiva-Nefrología y UCI. En estos servicios 3 o más de cada 4 tratamientos tienen una justificación o son interrumpidos por los propios tratantes cuando no hay necesidad de continuarlo.

\section{Discusión}

Los compuestos antimicrobianos corresponden a una de los fármacos más prescritos en pacientes hospitalizados $\mathrm{y}$, en diferentes evaluaciones, los porcentajes de uso inapropiado alcanzan altas proporciones $(\sim 30 \text { a } 60 \%)^{12}$. El programa de supervisión activa de tratamientos con imipenem-cilastatina demostró ser capaz de detectar usos inapropiados en diferentes servicios y unidades de nuestro hospital universitario. Para el período bajo análisis, aproximadamente 6 de cada 10 tratamientos con imipenem-cilastatina se justificaron, uno debió ser interrumpido por una indicación no apropiada, otros 2 fueron suspendidos por los propios médicos tratantes en algún momento durante los primeros 5 días desde su inicio y la fracción restante representó a aquellos pacientes dados de alta o fallecidos.

Una segunda visita de supervisión parece ser necesaria para aquellos pacientes en los cuales no se puede alcanzar una decisión durante el inicio del tratamiento. En nuestra experiencia, más de la mitad de los tratamientos interrumpidos fueron pesquisados en la segunda visita.

A pesar de que sólo $11,8 \%$ de los tratamientos iniciados fue considerado inapropiado, esta fracción representa un volumen considerable de tratamientos, que se expresa por el elevado número de días ahorrados de tratamiento y/o por la cantidad de frascos que no se utilizaron en el período. Más aún, este rendimiento se sumó al obtenido por los médicos tratantes que detuvieron los tratamientos en forma autónoma al programa de supervisión activa cuando no había una justificación. En porcentaje, esta cifra duplicó los tratamientos interrumpidos por el programa.

La capacidad de percibir un riesgo en la continuación del tratamiento con imipenem-cilastatina puede estar ligada a las actividades de capacitación sobre 
uso irracional de antimicrobianos que se desarrollan en este hospital universitario desde hace varios años, a la antigüedad y consistencia del programa (iniciado el año 2001) o a factores no esclarecidos. En cualquier caso, representan una ganancia estratégica y que también se ha reflejado en el programa de supervisión del uso de vancomicina, donde esta cifra se ha incrementado en forma progresiva (datos no mostrados). Las actividades de capacitación médica han sido útiles y eficaces en cambiar los hábitos de prescripción por parte de médicos de atención primaria o en aquellos que laboran en hospitales y se consideran un componente importante en el uso racional de antimicrobianos ${ }^{11}$.

Además de permitir una mejor calidad en la atención médica hospitalaria, el programa de supervisión activa de imipenem-cilastatina tuvo un claro impacto económico para el período analizado y por extrapolación, para todo un año. El ahorro para el período de 4 meses alcanzó los 3.524.133 pesos (6.777 dólares para una paridad cambiaria de 1 US $\$=520$ pesos) y para un año aproximadamente los 9.700 .000 pesos ( US \$18.630). El costo mensual del programa es bajo y no supera los 160.000 pesos ( 200 US\$) para cubrir gastos administrativos y de recursos humanos. Por cada dólar utilizado en supervisión sobre imipenem-cilastatina, se ahorraron aproximadamente 5,2 dólares. Ello implica que este programa puede ser extrapolado a otras instituciones de salud, aunque su impacto económico debe ser evaluado en terreno, ya que variaciones en la capacidad efectiva para interrumpir tratamientos inapropiados podrían menoscabar su relevancia económica.

Se debe tener presente que la estimación del ahorro anual presentada en este trabajo no es exacta y debe ser tomada sólo como un valor referencial debido al menos a tres factores identificados. En primer lugar, la dosis de imipenem-cilastatina que estaba siendo utilizadas en 2 de los casos interrumpidos, era excesiva (4 y 3 gramos, respectivamente) un factor que pudiera no repetirse en supervisiones futuras. En segundo lugar, la DDD de imipenem-cilastatina por días camas ocupadas durante los meses auditados fue en promedio menor a la de los meses no auditados, lo que podría subestimar el ahorro anual, ya que un mayor número de frascos podría haber sido suspendido en los otros meses. En tercer lugar, el programa de supervisión presentó fallas para efectivamente interrumpir la continuación del tratamiento en 4 de los 16 casos en los cuales éste fue objetado. La corrección de esta deficiencia podría permitir optimizar este sistema y ampliar sus beneficios en la calidad de atención de pacientes y lograr mayores ahorros.

No se exploró en este trabajo diferencias en los resultados clínicos obtenidos con los tratamientos al- ternativos en aquellos casos donde se cambió un tratamiento con imipenem-cilastatina. A priori se consideró una eficacia similar y por ende, el diseño del trabajo corresponde a un estudio de costo-minimización. Las razones para asumir esta equivalencia terapéutica obedecen a que todos los compuestos alternativos de la tabla 3 son utilizados como opciones cotidianas en el manejo de diferentes infecciones nosocomiales o comunitarias, corresponden a compuestos bactericidas y además se hayan respaldadas con estudios randomizados en varios casos. El desarrollo de un estudio de costo-efectividad para este tipo de reemplazo queda abierto a futuras indagaciones pero debe asegurar forzosamente algunas aristas como la existencia de grupos comparables en una diversidad de patologías y condiciones de base.

La evolución de los pacientes con reemplazo terapéutico fue favorable en la mayor parte de los casos y de los 3 pacientes que fallecieron, en al menos 2 casos, el deceso pareció más ligado a su condición terminal y no al fracaso del esquema alternativo.

La distribución de tratamientos inapropiados señala que el mal uso de este antimicrobiano puede ser focalizado, lo que resulta esencial para actividades de capacitación e intervención. Un indicador potencial de calidad en el uso de este antimicrobiano consideró la suma de los tratamientos aprobados y aquellos ya suspendidos por los médicos tratantes, permitiendo ordenar los servicios o unidades en forma jerárquica.

Los programas de restricción de uso de antimicrobianos han sido construidos con la premisa de controlar la emergencia de resistencia antimicrobiana entre bacterias asociadas a infección/colonización nosocomial o comunitaria. Sin embargo, este aspecto ha sido muy difícil de lograr o demostrar debido a las dificultades para diseñar estudios que permitan controlar la contaminación cruzada, a pesar de un favorable impacto sobre la resistencia, o que permitan evaluar apropiadamente este impacto a pesar de la estabilidad de los genotipos resistentes. Evaluaciones sistemáticas recientes no han encontrado una evidencia sólida para el resultado más esperado de los programas de restricción antimicrobiana, aunque algunas experiencias dan cuenta de la capacidad de estos programas para reducir el porcentaje de resistencia en aislados clínicos de Klebsiella pneumoniae o Enterobacter sp a moléculas específicas tales como aminoglucósidos y cefalosporinas de tercera generación ${ }^{11,16,17}$. El impacto para reducir tasas de resistencia entre aislados de bacilos gramnegativos no fermentadores o cocáceas grampositivas ha sido más difícil de lograr ${ }^{16,17}$. A pesar de que este aspecto no fue evaluado en nuestro estudio, no tenemos conocimiento de una publicación similar 
en Chile que haya evaluado el uso de antimicrobianos restringidos en un hospital.

Aunque limitado sólo al uso de imipenem-cilastatina, el porcentaje de uso apropiado observado en nuestro trabajo es concordante con datos previamente publicados para un conjunto de antimicrobianos ${ }^{12}$. En experiencias previas, los antimicrobianos restringidos son utilizados más racionalmente que aquellos sin restricción, y la opinión de un especialista en enfermedades infecciosas incrementa los porcentajes de uso apro-

\section{Anexo 1. Criterios de uso inapropiado de imipenem-cilastatina}

Manejo de Infecciones adquiridas en la comunidad, de cualquier tipo

Infecciones intrahospitalarias por bacilos gramnegativos que demuestran susceptibilidad a otros compuestos de eficacia equivalente

Manejo empírico de infecciones intrahospitalarias sin haber utilizado otras alternativas de amplio espectro en los 5 días precedentes (ejemplos: neumonía asociada o no asociada a ventilación mecánica de inicio precoz)

Tratamiento de pacientes con neutropenia febril u otras condiciones de inmunosupresión sin haber utilizado otros esquemas de amplio espectro en forma previa o con un cambio precoz a imipenem-cilastatina (24 horas) a pesar de la persistencia del cuadro febril

Tratamientos prolongados de infecciones asociadas a prótesis o cuerpos extraños sin remoción de ellos

Tratamiento basado en un urocultivo positivo a un bacilo gramnegativo multiresistente en presencia de cateterismo urinario permanente y sin manifestaciones clínicas de infección

Tratamiento basado en cultivos positivos de escaras de decúbito tomados en forma superficial, sin manifestaciones sistémicas de infección o sin intención terapéutica de mejorar la escara

Manejo del pie diabético sin cultivos de respaldo o basado en cultivos superficiales y sin la intención de revertir la condición isquémica de la extremidad piado $^{12}$. Mas aún, la remoción de una política de restricción por escasez de recursos humanos ha resultado deletérea con un gran efecto rebote en el consumo y costos de estos compuestos, ligado además a un deterioro en la calidad de atención ${ }^{18}$. Por otra parte, la restricción de compuestos antimicrobianos no está asociada a un deterioro en la calidad del paciente ya que no se asocia a un incremento en la mortalidad ${ }^{16}$.

\section{Resumen}

Imipenem-cilastatina es un compuesto de amplio espectro y de alto costo, reservado para el manejo de infecciones nosocomiales y que requiere ser restringido para evitar la emergencia de agentes resistentes a esta alternativa y para contener costos. Desde el año 2001 existe un programa de uso restringido y supervisión de este compuesto en nuestro hospital que permite aprobar o rechazar su uso. Se efectuó una auditoria de todas las supervisiones efectuadas durante marzoabril y septiembre-octubre del año 2004, totalizando 136 tratamientos. En términos globales, 58,1\% de los tratamientos fue considerado apropiado, $11,8 \%$ inapropiado y $20,6 \%$ ya había sido suspendido por médicos tratantes al momento de la visita de supervisión. La fracción restante incluye pacientes de alta, fallecidos o trasladados. Las interrupciones de tratamiento implicaron un ahorro de imipenem-cilastatina de 75 días, 362 frascos y 3.524 .133 de pesos (US 6,777) en los 4 meses de supervisión al incluir gastos en recursos humanos y costos administrativos.

\section{Referencias}

1.- García Moreno J, Dillon J R, Arroyave R, Maldonado A, Fich F, Salvo A, et al. Identification of penicillinase producing Neisseria gonorrhoeae in Chile during clinical and microbiological study of gonococcal susceptibility to antimicrobial agents. Genitourin Med 1987; 63: 6-12.

2.- Wolff M. Cambios epidemiológicos en las enfermedades infecciosas del país. Rev Méd Chile 2002; 130: 353-62.

3.- Prado V, Pidal P, Arellano C, Lagos R, San Martín O, Levine M. Multirresistencia antimicrobiana en cepas de Shigella $\mathrm{sp}$ en una comuna semi rural del área norte de Santiago. Rev Méd Chile 1998; 126: 1464 71.

4.- Trucco O, Prado V, Durán C. Red de vigilancia de resistencia antimicrobiana PRONARES: informe primer semestre 2001. Rev Chil Infect 2002; 19 (supl 2): S140-8.
5.- Fica A, Díaz P, López M, Zamorano J, Leiva A, Villalobos C. Altos niveles de resistencia en Escherichia coli causante de infecciones urinarias en una comunidad rural del área metropolitana. Rev Chil Infect 1999; 16: 120-6.

6.- Fica A, Pino A, Ramírez P, Brunel V, Triantafilo V. Susceptibilidad ante diferentes antimicrobianos en aislamientos de Escherichia coli de infecciones urinarias ambulatorias en Santiago. Revista Hosp Clínico Universidad de Chile 1998; 9: 232-7.

7.- Bello H, González G, Domínguez M, Valenzuela L, Zemelman C, Mella S, et al. Detection of extended-spectrum betalactamases produced by Chilean isolates of Klebsiella pneumoniae by two synergy methods. J Chemother 2004; 16: 312-4.

8.- Zemelman C, Bello H, Domínguez M, González G, Mella S, Zemelman R. Activity of cefepime, cefotaxime, ceftazidime, and aztreonam against extended-spectrum- producing isolates of Klebsiella pneumoniae and Escherichia coli from Chilean hospitals. Diagn Microbiol Infect Dis 2001; 40: 41-3.

9.- Marovac J, Campos MI. Enterococcus faecium resistente a vancomicina. Rev Méd Chile 2000; 128: 685-6.

10. Vlahovic-Palcevski V, Morovic M, Palcevski G. Antibiotic utilization at the university hospital after introducing an antibiotic policy. Eur J Clin Pharmacol 2000; 56: 97-101.

11.- Wilton P, Smith R, Coast J, Millar M. Strategies to contain the emergence of antimicrobial resistance: a systematic review of effectiveness and cost-effectiveness. J Health Serv Res Policy 2002; 7: 111-7.

12.- Erbay A, Colpan A, Bodur H, Cevik M A, Samore M H, Ergonul O. Evaluation of antibiotic use in a hospital with an antibiotic restriction policy. Int $\mathrm{J}$ Antimicrob Agents 2003; 21: 308-12.

13.- Keuleyan E, Gould I M. Key issues in 
developing antibiotic policies: from an institutional level to Europe-wide. European Study Group on Antibiotic Policy (ESGAP), subgroup III. Clin Microbiol Infect 2001; 7 (suppl 6): 16-21.

14.- Levin A S, Barone A A, Penco J, Santos M V, Marinho I S, Arruda E A G, et al. Intravenous colistin as therapy for nosocomial infections caused by multidrugresistant Pseudomonas aeruginosa and Acinetobacter baumannii. Clin Infect Dis 1999; 28: 1008-11.

15.- Fica A, Luppi M, Brito L, Castro A,
Abusada N. Impacto de un programa de supervisión sobre el uso de vancomicina en un hospital universitario. Libro de Resúmenes XVIII Congreso Chileno de Infectología, 23 al 26 de agosto del 2001, Pucón. Resumen CO8, pág 91.

16.- Sáez-Llorens X, Castrejón-De Wong M, Castaño E, De Suman O, Morós D, De Atencio I. Impact of an antibiotic restriction policy on hospital expenditures and bacterial susceptibilities: a lesson from a pediatric institution in a developing country. Pediatr Infect Dis J 2000; 19 ; 200-6.

17. - Rahal J J, Urban C, Horn D, Freeman K, Segal-Maurer S, Maurer J, et al. Class restriction of cephalosporin use to control total cephalosporin resistance in nosocomial Klebsiella. JAMA 1998; 280: 1233-7.

18.- Himmelberg C J, Pleasants R A, Weber D J, Kessler J M, Samsa G P, Spivey J M, et al. Use of antimicrobial drugs in adults before and after removal of a restriction policy. Am J Hosp Pharm 1991; 48: 1220-7. 